\title{
Fast sequence-based microsatellite genotyping development workflow
}

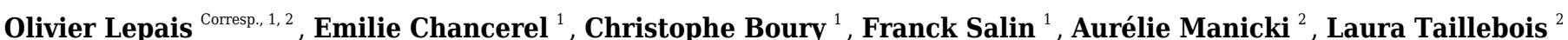
Cyril Dutech ${ }^{1}$, Abdeldjalil Aissi ${ }^{3}$, Cécile F E Bacles ${ }^{2}$, Françoise Daverat ${ }^{4}$, Sophie Launey ${ }^{5}$, Erwan Guichoux ${ }^{1}$

1 INRAE, Univ. Bordeaux, BIOGECO, Cestas, France

2 INRAE, Université de Pau et Pays de l'Adour, ECOBIOP, Saint-Pée-sur-Nivelle, France

3 LAPAPEZA, University of Batna 1 Hadj Lakhdar, Batna, Algeria

4 INRAE, EABX, Cestas, France

5 INRAE, Agrocampus Ouest, ESE, Ecology and Ecosystem Health, Rennes, France

Corresponding Author: Olivier Lepais

Email address: olivier.lepais@inrae.fr

Application of high-throughput sequencing technologies to microsatellite genotyping (SSRseq) has been shown to remove many of the limitations of electrophoresis-based methods and to refine inference of population genetic diversity and structure. We present here a streamlined SSRseq development workflow that includes microsatellite development, multiplexed marker amplification and sequencing, and automated bioinformatics data analysis. We illustrate its application to five groups of species across phyla (fungi, plant, insect and fish) with different levels of genomic resource availability. We found that relying on previously developed microsatellite assay is not optimal and leads to a resulting low number of reliable locus being genotyped. In contrast, de novo ad hoc primer designs gives highly multiplexed microsatellite assays that can be sequenced to produce high quality genotypes for 20 to 40 loci. We highlight critical upfront development factors to consider for effective SSRseq setup in a wide range of situations. Sequence analysis accounting for all linked polymorphisms along the sequence, quickly generates a powerful multi-allelic haplotype-based genotypic dataset, calling to new theoretical and analytical frameworks to extract more information from multi-nucleotide polymorphism marker systems. 


\section{Fast sequence-based microsatellite genotyping development workflow.}

2 Olivier Lepais ${ }^{1,2^{*}}$, Emilie Chancerel ${ }^{1}$, Christophe Boury ${ }^{1}$, Franck Salin ${ }^{1}$, Aurélie Manicki ${ }^{2}$, Laura Taillebois ${ }^{2}$,

3 Cyril Dutech ${ }^{1}$, Abdeldjalil Aissi ${ }^{3}$, Cecile F. E. Bacles ${ }^{2}$, Françoise Daverat ${ }^{4}$, Sophie Launey ${ }^{5}$, Erwan

4 Guichoux ${ }^{1}$.

5 IINRAE, Univ. Bordeaux, BIOGECO, F-33610 Cestas, France

62 INRAE, Université de Pau et Pays de l'Adour, ECOBIOP, F-64310 Saint-Pée-sur-Nivelle, France

$7 \quad{ }^{3}$ LAPAPEZA, University of Batna 1 Hadj Lakhdar, 0500 Batna, Algeria

$8{ }^{4}$ INRAE, EABX, Cestas Cedex F-33612, France

$9{ }^{5}$ INRAE, Agrocampus Ouest, ESE, Ecology and Ecosystem Health, F-35000 Rennes, France

${ }^{*}$ Corresponding author

Email address: olivier.lepais@inrae.fr

Abstract

Application of high-throughput sequencing technologies to microsatellite genotyping (SSRseq) has been shown to remove many of the limitations of electrophoresis-based methods and to refine inference of population genetic diversity and structure. We present here a streamlined SSRseq development workflow that includes microsatellite development, multiplexed marker amplification and sequencing, and automated bioinformatics data analysis. We illustrate its application to five groups of species across phyla (fungi, plant, insect and fish) with different levels of genomic resource availability. We found that relying on previously developed microsatellite assay is not optimal and leads to a resulting low number of reliable locus being genotyped. In contrast, de novo ad hoc primer designs gives highly multiplexed microsatellite assays that can be sequenced to produce high quality genotypes for 20 to 40 loci. We highlight critical upfront development factors to consider for effective SSRseq setup in a wide range of situations. Sequence analysis accounting for all linked polymorphisms along the sequence, quickly generates a powerful multi-allelic haplotype-based genotypic dataset, calling to new theoretical and analytical frameworks to extract more information from multi-nucleotide polymorphism marker systems. 
30

31

32

33

\section{Introduction}

In the age of high-throughput sequencing (HTS) technologies, and in comparison with SNP genotyping which is gaining momentum, application of HTS to microsatellite genotyping was until recently lagging behind. Traditional capillary electrophoresis-based microsatellite genotyping suffers from several drawbacks: homoplasy (alleles of identical size having different underlying sequence (Viard et al., 1998; Estoup, Jarne \& Cornuet, 2002)), time and cost consuming development and genotyping, low throughput, lack of automation and data standardization (Moran et al., 2006; Ellis et al., 2011). Yet, all of these limitations are linked to the fact that currently microsatellite genotyping relies on allele discrimination based on amplicon size assessed by capillary electrophoresis (De Barba et al., 2016) and do not hold true if microsatellite genotyping transitions to sequence-based genotyping. Previous direct comparisons of capillary electrophoresis and sequence-based microsatellite genotyping (called SSRseq thereafter) validated SSRseq as a reliable method (Darby et al., 2016; Vartia et al., 2016). Advantages of sequence-based over capillary electrophoresis-based microsatellite genotyping are significant. Direct access to allele sequence reveals additional polymorphisms that remain hidden when using only allele size to identify variation (Darby et al., 2016; Vartia et al., 2016; Šarhanová et al., 2018). Sequence data thus reduces allele homoplasy because alleles of the same size may contain molecular variation that does not translate into size variation such as SNP, indels masking variation in repeat number, or presence of two adjacent SSR motifs with complementary size variation (Darby et al., 2016). As a result, SSRseq offers refined genetic diversity estimation and population structure inference (Darby et al., 2016; Bradbury et al., 2018; Neophytou et al., 2018; Viruel et al., 2018; Layton et al., 2020).

Updating microsatellite genotyping to modern technologies remains important for several reasons. Firstly, some current scientific questions in ecology or evolutionary biology can be answered using a moderate number (e.g. a dozen (Harrison et al., 2013) to a hundred (Bradbury et al., 2018; Layton et al., 2020)) of highly polymorphic multi-allelic loci such as microsatellites. Secondly, variation in the number of repeated oligonucleotide motif is a unique kind of polymorphism with specific mutation mechanism and rate which in itself provides a complementary picture of genetic variation to nucleotide substitutions across populations (Haasl \& Payseur, 2011) and genomes (Willems et al., 2014). Thirdly, it becomes more and more obvious that microsatellite polymorphism is involved in numerous biological processes such as gene expression regulation and epigenetic mechanisms (Bagshaw, 2017; Sadd et al., 2018), and more generally in phenotypic variation (Xie et al., 2019) including human diseases (Gymrek, 2017; Hannan, 2018). Thus, while marker preference evolves through time with specific markers 
61

dominating the genotyping field over a period of time following technological advances (Schlötterer, 2004; Seeb et al., 2011), maintaining our capability to interrogate any kind of polymorphism in the context of rapid HTS technological advances is paramount and should be prioritized.

Studies published so far have explored specific technical or analytical aspects of SSRseq. Several bioinformatics approaches have been developed (Hoogenboom et al., 2016; Suez et al., 2016; Zhan et al., 2017; Barbian et al., 2018), different laboratory protocols tested (Vartia et al., 2016; Pimentel et al., 2018) and means to account for molecular variation on population genetic inference compared (Neophytou et al., 2018; Šarhanová et al., 2018; Viruel et al., 2018; Curto et al., 2019). Together, these studies explored numerous issues surrounding the technical and analytical advantages of SSRseq over traditional methods.

Here, we propose an integrative workflow for the development of a SSRseq analysis for application to non-model species. We apply this workflow to five species groups from families taken across phyla (Basidiomycota: Armillaria ostoyae; Angiosperms: Quercus faginea and Q. canariensis; Euarthropoda: Melipona variegatipes; Chordata: Alosa alosa, A. fallax and Salmo salar) that markedly differ in the amount of genomic data already available for them. We compare a broad range of possible development scenarios including sequencing of already optimized microsatellite assay traditionally genotyped on capillary sequencers, optimizing primers around already developed microsatellites, and developing microsatellite de novo from a range of genomic resources when available, or from newly generated low coverage random genome sequences for species without existing genomic resources. Building on our previous experience in development of highly multiplex microsatellite genotyping protocols (Guichoux et al., 2011; Lepais \& Bacles, 2011), we propose a streamlined approach with demonstrated application to groups of species with a wide range of genetic and evolutionary characteristics. We applied a microsatellite sequence data analysis pipeline to produce haplotypic data accounting for all polymorphisms detected in sequenced alleles, validated by extensive blind-repeat genotyping to estimate SSRseq error rates. We emphasize that efficient and powerful multipolymorphism haplotype-based genotyping approaches are easy to develop and apply, calling for new theoretical and analytical development to extract more information from multi-polymorphism haplotypes. 
91 Studied species, SSRseq development strategies and DNA isolation

92 We selected a range of species from different Kingdoms among biological models studied in our

93

94

95

96

97

98

99

100

101

102

103

104

105

106

107

108

109

110

111

112

113

114

115

116

117

118

119 laboratories (Table 1). For S. salar we took the most straightforward route by amplifying and sequencing microsatellites using previously developed primers. To develop a refined workflow, we chose species with different level of genomic resource availability to test alternative de novo microsatellite development strategies that are likely to cover a wide range of situations (Table 1).

\section{SSRseq using previously-developed primers}

The most straightforward approach to SSRseq microsatellite genotyping, i.e. based on sequence information from existing primers, was applied to S. salar using two marker selection strategies. In the first strategy, we selected 23 primers from a list of 81 microsatellites available for S. salar (O'Reilly and Wright 1995; Slettan et al. 1996; Ozaki et al. 2001; Rexroad et al. 2001; Gilbey et al. 2004; Paterson et al. 2004; King et al. 2005; Vasemägi et al. 2005; Thorsen et al. 2005; Yano et al. 2013, see details in Supporting Table S1). Selection criteria included allele size smaller than 300 bp to ensure that sequencing reads can span the entire allele length including library construction and absence of sequences complementarity between primers tested using Multiplex Manager (Holleley \& Geerts, 2009). In the second strategy, we chose to sequence a set of 15 microsatellites (Supporting Table S1) that are routinely amplified in a single multiplexed PCR and genotyped using standard capillary electrophoresis (Bacles et al., 2018; Lepais et al., 2017).

\section{SSRseq with microsatellite (re)development}

Genomic resources for microsatellite (re)development

For the other species, microsatellites primers were either re-designed or developed de novo from various genomic resources (Table 1, Figure 1 top panel).

For Quercus sp., primers were re-design primers in flanking regions of existing microsatellite markers to optimize multiplex amplification and sequence interpretability while taking advantage of already validated microsatellite markers. We extracted primer sequences from 259 polymorphic and mapped EST-derived (Durand et al., 2010) and 35 genomic microsatellites (Steinkellner et al., 1997; Kampfer et al., 1998)). The primer sequences were mapped on the Q. robur reference genome (Plomion et al. 2018, GenBank accession GCA_003013145.1) using bowtie 2 v2.3.4.1 (Langmead \& Salzberg, 2012) and genomic sequence spanning from $200 \mathrm{bp}$ downstream of the forward primer to $200 \mathrm{bp}$ upstream of the

Peer) reviewing PDF | (2019:12:44260:1:1:NEW 14 Mar 2020) 
120

121

122

123

124

125

126

127

128

129

130

131

132

133

134

135

136

137

138

139

140

141

142

143

144

145

146

147

148

149

reverse primer position were extracted using bedtools v2.25.0 (Quinlan, 2014) resulting in 294 sequences used as genomic resource to design new primers.

For Alosa sp., we used sequences obtained from a Roche 454 GS-FLX sequencing run on a microsatelliteenriched DNA library (Rougemont et al., 2015) following the method described in Malausa et al. (2011).

For $A$. ostoyae, we used the reference genome sequence as genomic resource to identify microsatellites loci (Sipos et al. 2017, GenBank accession GCA_900157425.1).

Finally, no genomic resources were available for $M$. variegatipes. We therefore used DNA from one individual to construct a whole-genome sequencing library using Illumina TruSeq DNA kit. The resulting library was sequenced on an Illumina MiSeq flowcell using v3 2×300 pb paired-end sequencing kit. Mothur software v1.39.5 (Schloss et al., 2009) was used to assemble paired reads and keep paired reads with a minimum overlap of $100 \mathrm{bp}$ without mismatch. We randomly subsampled 500,000 reads from the resulting 6.74 million paired reads for subsequent microsatellite identification, because a few hundreds of thousands of random sequences are sufficient to identify thousands of microsatellites (Castoe et al., 2010; Lepais \& Bacles, 2011; Curto et al., 2019).

\section{De novo microsatellite development or primer re-design}

The command line version of QDD pipeline v 3.1 (Meglécz et al., 2010, 2014) was run on either i) a reference genome sequence, ii) a set of low coverage random sequences or iii) sequence extracted around already characterised microsatellite loci (Table 2) , to detect sequences containing microsatellites, identify good quality sequence (singletons and consensus) from problematic sequences (sequences showing low complexity, minisatellites or multiple BLAST hits with other sequences) and design primer pairs flanking the identified microsatellites (Figure 1). QDD pipeline was run with default parameters, except for the primer design step (pipe3) where parameters were stringently defined in order to improve their capacity to be amplified jointly in a single multiplexed PCR (Qiagen Multiplex kit handbook; Lepais \& Bacles 2011): primer optimal size was set to 25 nucleotides (min: 21, max: 26), optimal annealing temperature to $68^{\circ} \mathrm{C}\left(\min : 60^{\circ} \mathrm{C}\right.$, max: $\left.75^{\circ} \mathrm{C}\right)$ with a maximal difference of $10^{\circ} \mathrm{C}$ between primers of a same pair and optimal percentage of cytosine and guanine of $50 \%$ (min: $40 \%$, max: $60 \%)$. In addition, PCR product size was set between 120 and $200 \mathrm{bp}$ to be compatible with a wide range of sequencing platforms and to produce robust genotyping assays that can be used to analyse degraded or low quantity DNA samples. QDD analysis results in a large number of candidate loci with designed 
150 primer pairs from which a restricted number of loci can be selected (Figure 1). At the exception of

151 Quercus sp. where a restricted set of input sequences necessarily limited choice among resulting

152 candidate microsatellites, several quality criteria were used to select 60 microsatellites among hundreds

153 to thousands candidates for further testing. We followed recommendations of Meglécz et al. (2014) to

154 prioritize primer pairs with increased likelihood of amplification success by selecting microsatellite from

155 singletons and not from consensus sequence, with pure repeat instead of compound motifs, showing at

156 least $20 \mathrm{bp}$ between the primers and the repeat motif, and with flanking region showing high complexity

157 (e.g. primer pairs from the design group A following QDD terminology: no minisatellite, no other

158 microsatellite in the flanking region, no homopolymer in the flanking region or the primer). In addition,

159 we further selected microsatellites with the highest number of repeats to increase the probability of

160 selecting polymorphic loci, avoided motif that can form hairpin such as AT repeats and when possible

161 included a variety of di-, tri- and tetra nucleotide repeats.

162 Primer modification and simplex amplification tests

163 For Ion Torrent sequencing (Table 2), tag A 5'-GCCTTGCCAGCCCGCTCAG-3' was add to the 5' end of each

164 forward primer and tag B sequence 5'-GCCTCCCTCGCGCCA-3' (Margulies et al., 2005; Blacket et al.,

165 2012) was added to the $5^{\prime}$ end of each reverse primer. For Illumina sequencing (Table 2), specific tags 5'-

166 TCGTCGGCAGCGTCAGATGTGTATAAGAGACAG-3' and 5'-

167 GTCTCGTGGGCTCGGAGATGTGTATAAGAGACAG-3' were added to the 5' end of the forward and reverse

168 primer sequences respectively. Designed primers were tested for potential primer dimer formation

169 using Primer Pooler (Brown et al., 2017). Primer pairs showing a deltaG lower than $-6 \mathrm{kcal} / \mathrm{mol}$ are likely

170 to form dimer and result in poor amplification in a multiplexed PCR. For locus involved in significant

171 interactions, alternative primers were selected or in absence of alternative, another locus was selected

172 from the candidate list. Oligonucleotides were ordered in a plate format from Integrated DNA

173 Technologies with standard desalt purification at a final concentration of $100 \mu \mathrm{M}$. Primer pairs were

174 tested using simplex amplification of one individuals per species using Qiagen Multiplex kit in a final

175 volume of $10 \mu \mathrm{L}$ and with a final concentration of each primer of $0.2 \mu \mathrm{M}$. Amplification conditions

176 consisted of an initial denaturation step at $95^{\circ} \mathrm{C}$ for 15 minutes, followed by 35 cycles consisting of

177 denaturation at $95^{\circ} \mathrm{C}$ for $20 \mathrm{~s}$, annealing at $59^{\circ} \mathrm{C}$ for $60 \mathrm{~s}$ and extension at $72^{\circ} \mathrm{C}$ for $30 \mathrm{~s}$, and a final

178 extension step for 10 minutes at $72^{\circ} \mathrm{C}$. Amplicons and $1 \mathrm{~Kb}$ size standard were then loaded on a $3 \%$

179 agarose gel containing GelRed or SyberSafe dye and migrated at 100v for 15 minutes. Each locus was

180 screened under UV light for positive amplification with a clear band at the expected size.

Peer] reviewing PDF | (2019:12:44260:1:1:NEW 14 Mar 2020) 
183

184

185

186

187

188

189

190

191

192

193

194

195

196

197

198

199

200

201

202

203

204

205

206

207

208

209

210

211

From 192 to 960 individuals were analyzed depending on the taxa considered including from 46 to 156 repeated individuals to check the reproducibility of the method (Table 2). For each of the taxonomic groups, a three-round multiplex PCR approach was used to amplify all loci simultaneously and improve amplification homogeneity and thus coverage of sequence between loci (Chen et al., 2016). In the first round, a multiplexed PCR including all selected locus primers was performed (Figure 1, Supporting Table S1 for locus characteristics including primer sequences). PCR amplification were carried out in 96-well plates in a final volume of $5 \mu \mathrm{L}$ or $10 \mu \mathrm{L}$ using Qiagen Multiplex kit, $0.05 \mu \mathrm{M}$ of each forward and reverse tailed primers and about $40 \mathrm{ng}$ of template DNA (depending on the species, $1 \mu \mathrm{L}$ of undiluted or diluted isolated DNA). PCR cycles were performed on Applied Biosystems 2720 or Verity thermocyclers and consisted of a denaturing step of $5 \mathrm{~min}$ at $95^{\circ} \mathrm{C}$ followed by 20 cycles of $95^{\circ} \mathrm{C}$ for $30 \mathrm{~s}, 59^{\circ} \mathrm{C}$ for $180 \mathrm{~s}$ and $72^{\circ} \mathrm{C}$ for $30 \mathrm{~s}$ (Qiagen Multiplex kit handbook; Lepais \& Bacles 2011). In the second round, additional Taq polymerase added with the aim to use remaining primers completely. The PCR mixture of a final volume of 5 or $10 \mu \mathrm{L}$ consisted of 2.5 or $5 \mu \mathrm{L}$ of Qiagen Multiplex kit, 1.5 or $3 \mu \mathrm{L}$ of undiluted amplicon and 1 or 2 $\mu \mathrm{L}$ of water. The PCR cycles were identical as in the first round. The third round is the indexing PCR (Figure 1) that add lon Torrent or Illumina sequencing adaptors and barcodes used to assign each sequence to an individual. For lon Torrent sequencing, we used 106 different barcodes resulting in a total of 960 barcode combinations. For Illumina sequencing, we used the Nextera XT index set allowing for 384 barcode combinations. The PCR mixture of a final volume of $10 \mu \mathrm{L}$ consisted of Qiagen Multiplex kit Master Mix, $0.5 \mu \mathrm{M}$ of sequencing platform-specific adaptor and $5 \mu \mathrm{L}$ of undiluted amplicon resulting from the second PCR round. PCR cycles consisted of a denaturing step of $5 \mathrm{~min}$ at $95^{\circ} \mathrm{C}$ followed by 15 cycles of $95^{\circ} \mathrm{C}$ for $30 \mathrm{~s}, 59^{\circ} \mathrm{C}$ for $90 \mathrm{~s}$ and $72^{\circ} \mathrm{C}$ for $30 \mathrm{~s}$ and a final extension step of $68^{\circ} \mathrm{C}$ for 10 minutes. Amplicons from the 96 wells within a plate were pooled together in an Eppendorf tube, and purified with 1.8X Agencourt AMPure XP beads (Beckman Coulter, UK). Quality check and quantification were done using Agilent Tapestation D1000 kit and Qubit fluorometric system (Thermo Fisher Scientific), and quantified using Kapa libraryquantification kit in a Roche LightCycler 480 quantitative PCR. The resulting two to ten pools were pooled in equimolar concentration and sequenced using an lon Torrent PGM i316 chip or Illumina MiSeq flowcell using nano or v2 2×250 bp paired-end sequencing kit (Table 2) at the Genome Transcriptome Facility of Bordeaux.

\section{Bioinformatics data analysis}




\section{Sequence preparation}

213 After sequence demultiplexing and adaptor trimming using a sequencer platform built-in software,

214 quality was controlled using FastQC (http://www.bioinformatics.babraham.ac.uk/projects/fastqc/) and

215 reads shorter than $70 \mathrm{bp}$ were removed using cutadapt (Martin, 2011). When paired-end sequencing

216 was used, paired reads were assembled into contigs using pear (Zhang et al., 2014) with the default

217 scoring method based on assembly score (allowing for mismatch and accounting for base quality

218 scores), a minimum overlap of $50 \mathrm{bp}$ and a maximum assembled sequence length of $450 \mathrm{bp}$. For Alosa

219 sp., reverse reads quality was generally poor, therefore, only the forward read was used as its length

220 (250 bp) encompasses the whole length of the sequenced loci (max. $200 \mathrm{bp}$ ). In some cases,

221 microsatellite amplicons from several species were pooled prior to PCR indexing so that two individuals

222 from two species shared an identical barcode combination and were sequenced in a single run. Forward

223 primer sequences of species-specific loci were used to sort sequences belonging to different species into

224 different fastq files using fqgrep tool (https://github.com/indraniel/fqgrep) allowing for one mismatch.

225

226

227

228

229

230

231

232

233

234

235

236

237

238

239

240

241

\section{Converting microsatellite sequences to genotypes}

We used FDSTools v1.1.1 pipeline (Hoogenboom et al., 2016) to identify sequences corresponding to the microsatellite alleles and call genotypes for each individual (Figure 1). This analytical tool was chosen because it accounts for any kind of polymorphism detected across the analysed sequences (including variation in the number of repeated motifs, SNP or indels) while integrating specific tools to detect true allele from stutter mutation introduced during amplification that are typical of microsatellite markers.

First, tssv (Anvar et al., 2014) matches primer sequences, allowing for $8 \%$ of mismatch, to identify sequences originating from each locus and count the occurrence of each unique sequence found for each locus for each individual (Figure 1). Then, Stuttermark uses the number of repeats of the microsatellite motif and the coverage of each unique sequence to flag unique sequences as potential allele, stutter resulting from slippage mutation during PCR and erroneous sequences (Figure 1). Finally, Allelefinder calls one or two alleles among the most abundant sequences flagged as potential alleles by Stuttermark (Figure 1). Following the FDSTools analysis, several custom-made bash routines were used to format the tabulated genotypic table, compare genotypes from repeated individuals to estimate locus specific allelic error rate defined by the number of allele mismatches between replicated genotypes divided by the number of alleles compared. In addition, allele level information was extracted including allele sequences, three-digit code used for genotype annotation, number of occurrence across

Peer) reviewing PDF | (2019:12:44260:1:1:NEW 14 Mar 2020) 
242 individuals and allele length. Locus characteristics such as missing data rate and number of alleles are

243 also summarized across the analysed individuals (Figure 1). All these bioinformatics steps have been

244 embedded into a single bash script (SSRseq_DataAnalysis_ParametersComparison.sh available at

245 https://doi.org/10.15454/HBXKVA) that allow to modify key analytical parameters (Supplemental

246 Information S1) to evaluate their effect on the quality of the genotypic call (number of detected alleles,

247 error and missing data rates).

248 Two analytical strategies were compared. In the first strategy (called FullLength thereafter), all variation

249 identified between primers was considered as a haplotype irrespectively of the nature of the

250 polymorphism because all polymorphisms are physically-linked to each other in reads that encompass

251 the whole locus. The FullLength strategy may be too complex for some loci or species showing high

252 levels of polymorphism. In the second strategy, the analysis was therefore restricted to the repeat motif

253 only (strategy called RepeatFocused thereafter). In this case, primer and flanking sequences surrounding

254 the repeated motif are indicated in the primer sequence field of the FDSTools input file. The tssv step

255 then extracts the sequence corresponding to the repeat motif region (still allowing for $8 \%$ of mismatch

256 which will accommodate flanking sequence polymorphism) to perform subsequent genotypic call with

257 Stuttermark and Allelefinder as described above. As the analytical approach used by FDSTools is based

258 on counting coverage of unique sequences, any variation identified within the repeated motif region,

259 including variation in repeated motif number, SNP and indel within the motif region, will still be

260 accounted for when defining alleles. While this RepeatFocused strategy may be more robust due to the

261 shorter length of sequence analysed, it should identify a smaller number of alleles compared to the

262 FullLength strategy.

\section{Comparing analytical approaches}

264 For each locus, we determined the best analytical strategy using the following criteria by order of

265 importance: estimated allelic error, amount of missing genotypes and number of detected alleles. Loci

266 that showed more than $6 \%$ of allelic error or more than $50 \%$ of missing data across individuals (within

267 each species group) were flagged as failed and removed from further inspection. These arbitrary

268 thresholds have been chosen to remove bad quality loci while conserving moderate quality loci (see

269 Supporting Table S1 for locus specific missing data and allelic error rates). Decreasing these thresholds

270 will have resulted in the removal a handful of loci for each species, the majority of loci having low

271 genotyping error and missing data (Supporting Table S1). For each species and analytical strategy, we

272 recorded the number of genotyped loci, mean allelic error and missing data rate and the total number of 
273 alleles (haplotypes) across loci. We then determined the best overall approach for each locus and used it

274 to genotype each locus generating a final Combined genotypic dataset for each species using a specific

275 bash script (SSRseq_DataAnalysis_FinalGenotyping.sh available https://doi.org/10.15454/HBXKVA).

276 Finally, the overall development success rate was computed for each species by dividing the number of

277 reliable loci by the number of loci included in the multiplexed PCR.

\section{Gain from sequence information}

279 The number of identified alleles based on sequence information (haplotypes) was compared to the 280 number of alleles differing in amplicon length only for all analysed loci to assess the gain of information obtained by using sequence data and estimate size homoplasy. We investigated further the nature of the detected polymorphism by counting, for each locus, the number of variations in the number of repeats, SNP and indels in the repeated motif and the flanking regions that differ between haplotypes.

\section{Results}

\section{Sequence-based genotyping of previously developed microsatellites}

Attempts to genotype previously developed microsatellites in S. salar either from a new combination of 23 loci or a routinely-used multiplex of 15 loci resulted in a low number of reliable loci genotyped (Table 2). The overall success rate (the percentage of reliable loci over the number of loci amplified in the multiplexed PCR) ranged from $39 \%$ to $47 \%$ and the number of reliable loci from 7 to 10 (Table 2). Moreover, the quality of the generated genotypic datasets is relatively low with a rate of missing data and allelic error above $10 \%$ and $1 \%$ respectively (Figure 2 ). The sequences produced on the PGM Ion Torrent platform resulted in the lowest genotypic data quality (Figure 2). The same genotyping protocol sequenced on the Illumina MiSeq platform produced higher genotypic quality with lower missing data and allelic error rates (Figure 2) in spite of a 2.3 times lower mean coverage per sequenced locus per individual (Table 2). The lowest performance of the lon Torrent platform is due to the higher sequencing error rate linked to spurious insertion-deletion around homopolymer tracts. This results in a waste of sequencing reads, increasing noise (e.g. erroneous singletons: unique sequence with a coverage of one or a very few reads), at the expense of sequence exactly matching the true alleles. The Illumina MiSeq sequencing platform was thus used for subsequent analyses in other species. 
303 In contrast, overall success rate of de novo microsatellite development ranged from $67 \%$ to $86 \%$ (Table

2). Given the high number of candidate microsatellites typically identified from high-throughput sequencing or reference genome sequence, we were able to screen as much as 60 new loci, and combined most of them (from 28 to 60 ) in a single multiplexed PCR for amplification (Table 2). As a result, the final number of reliable loci was consistently high amounting to 24 for Alosa sp., 38 for $A$. ostoyae, 39 for M. variegatipes and 40 for Quercus sp. (Table 2). All these protocols produced high quality genotypic dataset, with low missing data (2.6\% for Quercus sp., 6.8\% for Alosa sp., 5.4\% for M. variegatipes) at the exception of $A$. ostoyae (20.7\%) and low allelic error rates as estimated based on blind-repeat genotyping ( $0.4 \%$ for Quercus sp., $0.6 \%$ for Alosa sp., $0.9 \%$ for $M$. variegatipes and $0.7 \%$ for A. ostoyae). For Alosa sp. and Quercus sp. all reliable loci were found to be transferable between species.

We explored the effect of sequence coverage on genotypic data quality on Alosa sp. by sequencing the same set of 28 microsatellites amplified in 156 individuals using one, two or three Illumina MiSeq nano flowcells (Table 2). The resulting increased coverage, from 95 to 198 and 267 sequences respectively per locus per individual (Table 2), recovers more data for those highly degraded DNA samples. First, increasing the coverage from 95 to 198 sequences by locus by individual detected three additional loci, while a further increase in coverage failed to recover additional locus (Table 2). Second, the missing data rate linearly decreases with the increase in coverage, from $16.4 \%$ to $9.0 \%$ and $6.8 \%$ with 95,198 and 267 sequences per locus per individual respectively (Supporting Material Figure S1). It is worth noting that the allelic error rate is not affected by genome coverage, as it varied only slightly between $0.5 \%$ and $0.7 \%$ without any correlation to coverage (Supporting Material Figure S1). A significant result is that even in conditions when only highly degraded DNA templates are available, reliable genotypic data can be obtained with moderate coverage, while increasing coverage will reduce missing data but not genotyping error rate.

\section{Whole sequence VS repeated motif polymorphism analysis}

Focusing the analysis on the repeated motif slightly increased the number of reliable loci and tends to 
331

332

333

334

335

336

337

338

339

340

341

342

343

344

345

346

347

348

349

350

351

352

353

354

355

356

357

358

359

360

361

polymorphism detected between the PCR primers resulted in a higher mean number of allele per locus, at the expense of slightly higher missing data and allelic error rates (Figure 3). Interestingly, 17\% of the loci can be analysed reliably using either the FullLength or the RepeatFocused analytical approach. Thus combining analytical strategies by selecting the best approach for each locus resulted into an optimized dataset (Figure 3). Even for loci with reliable genotypes irrespectively of the analytical approach chosen, selecting the one that produces the best quality data (in terms of number of alleles, missing data and error rate) leads to an improved dataset quality. This combined strategy results in recovering the highest number of loci and alleles while keeping missing data and allelic error rates at the lowest (Figure 3).

\section{Types of polymorphism detected across species}

While most common population genetics applications do not necessitate to characterize the nature of the polymorphism differentiating alleles, the main advantage of sequence data (in addition to analysing a much higher number of loci) is to be able to identify allelic variation that does not translate into size variation, i.e. the only variation that is detected when using classical electrophoretic approaches. Across species, the proportion of alleles would have remained undetected by capillary electrophoresis (size homoplasy) ranging from $6 \%$ for $M$. variegatipes, $11 \%$ for S. salar, 14\% for Alosa sp., 35\% for Quercus sp. and $53 \%$ for $A$. ostoyae (Table 3 ). Conversely, the increase in the proportion of allele detected by accessing sequence data ranges from $6 \%$ for $M$. variegatipes, $13 \%$ for $S$. salar, $16 \%$ for Alosa sp., $56 \%$ for Quercus sp. and $113 \%$ for $A$. ostoyae (Table 3). Indeed, beside variation in repeat number, we identified numerous SNP and indel either in the flanking sequence or in the repeat motif itself (Figure 4, Table 3). In fact, additional polymorphism beyond variation in repeat number was the rule rather than the exception (Table 3). However, differences in the proportions of the type of the detected polymorphism were found between species (Figure 4). While repeat number variation represented more than $80 \%$ of the polymorphism detected in S. salar, Quercus sp., Alosa sp. and M. variegatipes, SNP in the flanking sequence was the most frequent polymorphism for $A$. ostoyae representing $49.8 \%$ of the variation, much higher than variation of repeat number estimated at $29.8 \%$ (Figure 4). For A. ostoyae, SNP in the repeat motif and indel in the flanking sequence also represent a significant proportion of the polymorphism detected ( $12.6 \%$ and $6.5 \%$ respectively). Quercus sp. were characterised by SNP both within the repeat motif (7.5\%) and the flanking region (9.3\%). The second most common polymorphism for Alosa sp. was SNP in the repeat motif (8.5\%) and for M. variegatipes SNP in the flanking region (5.5\%). For S. salar, variation in the number of motif was much more frequent than for other species 
$362(93.0 \%)$ compared to other polymorphisms that represent a marginal proportion of the variation (less 363 than 3\% each).

\section{Discussion}

366

367

368

369

370

371

372

373

374

375

376

377

378

379

380

381

382

383

384

385

386

387

388

389

390

While relying on previously developed microsatellite assays is far from optimal, we found that primer redesign around known locus or de novo microsatellite development based on strict criteria gives successful single highly multiplexed PCR amplification and sequencing for 20 to 40 loci. However, key initial factors need to be considered for efficient SSRseq setup.

\section{Not all roads lead to Rome: navigating pitfalls when adopting HTS for microsatellite genotyping}

Our preliminary attempts to apply SSRseq using previously developed microsatellite primers or capillarybased multiplexed microsatellite genotyping protocols clearly failed to produce reliable genotypic data for a sufficient number of loci. In the best case scenario, sequencing of 15 microsatellites in Salmo led to the reliable genotyping of 7 loci with $15.6 \%$ of missing data and $1.19 \%$ of allelic error, a result far worse than the high quality dataset obtained for the same multiplex using traditional capillary-electrophoresis of 14 loci with $0.5 \%$ missing data and $0.35 \%$ allelic error (Bacles et al., 2018). Similar trends were observed in previous studies that validated the use of HTS to genotype microsatellites and relied on previously developed primers: the generated datasets were characterised by high levels of missing data (up to 45\%, (Vartia et al. 2016)) or low number of genotyped loci (7 to 8, (Suez et al. 2016; Barbian et al. 2018)). Two characteristics are problematic for SSRseq from microsatellites initially developed for capillary electrophoresis-based genotyping. First, high variability in locus length and primer characteristics leads to heterogeneous amplification intensity and sequencing coverage across loci. Indeed, efficient multiplexed PCR necessitates careful primer design using strict criteria (Guichoux et al., 2011; Lepais \& Bacles, 2011). In addition, the need for variable locus length for optimal multiplexing without allele size range overlap in capillary-based electrophoresis becomes unnecessary for sequencing, because same size loci can be reliably identified simply based on primer sequences. Secondly, starting from a limited number of loci (e.g. 10-20 typical of capillary electrophoresis-based microsatellite genotyping approaches) results in a handful of reliable loci that may be too low for downstream applications. This conclusion agrees well with a previous study developing SSRseq in S. salar, where only one out of six (17\%) of previously developed loci was successfully integrated into the 
391 final panel, compared with a $26 \%$ success rate for newly developed primers (Bradbury et al., 2018).

392 Adapting previously developed loci in the same species resulted in a success rate of about $45 \%$ in our 393 case (7 out of 15 and 10 out of 23 reliable loci), but de novo development in other species was much 394 more successful with a success rate of $75 \%$ on average. We did not retrospectively apply the refined 395 SSRseq development approach for S. salar to confirm it is also working on this species because it would duplicate recent protocol developed for this species (Bradbury et al., 2018) with limited usefulness for the scientific community. Nevertheless, our result clearly show that not relying of previous primer design is of primary importance for the success of reliable SSRseq protocol development.

Workflow for efficient SSRseq development in non-model species

400

401

402

403

404

405

406

407

408

409

410

411

412

413

414

415

416

417

418

419

420

421

We propose here a workflow for developing new SSRseq approaches and we demonstrated its efficiency for a range of species with different level of genomic resource availability. Starting from a reasonable number of candidate loci that were identified using readily available or newly generated genomic resources, resulted in an average of $75 \%$ of the loci included in the PCR multiplex generating reliable genotypic data. This result compares favourably with previous studies where success rates were lower (47\% (Farrell et al., 2016), 53\% (Neophytou et al., 2018)) or similar (78\% (Tibihika et al., 2018)) when developing about 20 to 40 loci from a moderate number of candidate loci. However, extensive screening of numerous markers resulted in a much lower success rate: 101 validated loci from 385 tested (26\% success rate (Bradbury et al., 2018)) or 43 validated loci from 448 locus tested (10\% (Zhan et al., 2017)). Note however that leveraging extensive whole genome resequencing data can result in significant improvement of development success by targeting polymorphic microsatellites with perfect repeat motif and invariant flanking sequences (Yang et al., 2019). In this study, we propose a balanced approach for effective development that relies on simple amplification tests of a limited number of carefully selected candidate loci. Locus validation is then made when sequencing the final set of loci in a way that the validation step, based on the analyse of blind-repeat of at least 48 individuals, jointly generates useful genotypic data (for additional individuals included in the sequencing run, Table 2). In addition, most of the ordered primers will be validated and integrated in the final set of reliable loci which greatly minimise development costs. The workflow is also flexible in terms of number of loci chosen for analysis: if a higher number of loci is required; additional sets of 60 loci could be selected from the candidate locus list and amplified in separated PCR multiplexes that can be pooled together before PCR indexing leading to an effective way to genotype additional loci (Bradbury et al., 2018). We did not test multiplexing more than 60 loci in a single PCR, but we did not see difficulties in doing so 
422 (Campbell, Harmon \& Narum, 2015). In such a situation, careful primer design with strict criteria and

423 control for primer interactions will be key to increase multiplexed amplification success.

424 The proposed workflow for SSRseq development minimized laboratory steps and analytical

425 optimizations. We chose to start from a moderate number of loci, designed to maximize their

426 compatibility and sequence interpretability, and remove any locus that failed to amplify, produce

427 interpretable or repeatable genotypes. This strategy avoids tedious optimization and reduce the number

428 and complexity of laboratory steps necessary to produce genotypes. Admittedly, additional DNA or

429 amplicon clean up or normalisation might improve sequence quality and coverage across individuals and

430 loci. However, we chose to keep the laboratory procedure as simple as possible and compensate the

431 increase in amplification heterogeneity by additional sequence output resulting in sufficient coverage

432 (220 reads/loci/individuals on average) to obtain a nearly complete genotypic dataset (generally $5 \%$ of

433 missing data excluding the atypical case of Armilaria species). Moreover, increasing the number of

434 laboratory steps inflates the risk of handling error and subsequently of genotyping error (Vartia et al.,

435 2016). In this respect, highly multiplexed PCR are a useful technique to reduce laboratory steps and

436 potential associated errors in addition to saving time and cutting costs. Additional tests not presented

437 here in addition to results from previous studies (De Barba et al., 2016; Zhan et al., 2017; Bradbury et al.,

438 2018; Tibihika et al., 2018) showed that the two-stage multiplex amplification prior to PCR indexing that

439 was used to increase amplification homogeneity across loci is not necessary: high coverage is still

440 efficient to compensate the increase in amplification heterogeneity when using a single multiplex PCR

441 step for locus amplification.

442 We chose to rely on the set of 384 Illumina barcodes combinations because we found it to fit well with

443 the output of the MiSeq sequencing platform when analysing from 20 loci to 300 loci depending on the

444 type of flow cell used. However, studying more than 384 individuals from a single species necessitates

445 either several MiSeq runs (Bradbury et al., 2018) or custom made dual-indexing strategies, as was

446 successfully performed for the lon Torrent PGM run (960 barcode combinations used) or in previous

447 studies using the MiSeq platform (960 and 1024 barcode combinations (Farrell et al., 2016; Zhan et al.,

448 2017)).

449 Finally, including repeated individuals is of paramount importance to assess the reliability of the

450 produced genotypic data for each locus. This procedure aims to be best practice in capillary

451 electrophoresis-based microsatellite genotyping (Hoffman \& Amos, 2005; Pompanon et al., 2005;

452 Guichoux et al., 2011) but is even more important in SSRseq. Indeed, not all sequenced loci produced 
453 reliable genotypic data. It is thus necessary to be able to identify and exclude loci producing high

454 genotyping errors. At the bioinformatics analysis stage, selecting the best analytical strategy for each

455 locus is an easy task that improve the number of reliable loci and decrease the genotyping error rate.

456 However, a few loci will still show high genotyping error rate due to low coverage or complex

457 polymorphism patterns and should be excluded from the final genotypic dataset. Exploratory analyses

458 testing a much wider number of parameters resulted in limited success in increasing the final number of

459 reliable locus. Extensive parameter testing is a tedious task requiring high computation time with only

460 minor improvement for the final genotypic dataset quality and is not worth the extra effort as long as a

461 sufficient number of loci have been included in the genotyping panel. Furthermore, additional analyses

462 using alternative microsatellite sequences analysis tools such as Megasat (Zhan et al., 2017) and

463 MicNeSs (Suez et al., 2016) also lead to the conclusion that all loci cannot be analysed reliably using a

464 single set of parameters. Thus, we stress the importance to include a significant number of repeated

465 individuals (at least 48) in the first analysis of a new SSRseq panel, irrespective of the bioinformatics data

466 analysis strategy used, to (1) coarsely optimize analysis parameters for reliable loci, (2) quantify

467 genotyping error rate and (3) identify and exclude unreliable loci. Such procedure has been

468 implemented only in a limited number of previous studies describing SSRseq methods (6 out of 14

469 published studies thus far (De Barba et al., 2016; Zhan et al., 2017; Bradbury et al., 2018; Barbian et al.,

470 2018; Šarhanová et al., 2018; Viruel et al., 2018)) but should be generalized. Downstream biologically-

471 informed statistical analyses to verify that the loci comfort to Hardy-Weinberg equilibrium, detect the

472 presence of null-alleles and estimate genotyping error rate based on sibship inference in natural

473 population or known pedigree (Wang, 2017) should then be applied to further validate the obtained

474 genotypes.

\section{Implications of haplotype based genotyping}

476 We took advantage of the fact that reads span across entire loci to analyse all linked and phased

477 polymorphisms encountered using the FDSTools pipeline (Hoogenboom et al., 2016). This haplotype

478 approach differs from the methods implemented in other sequence-based microsatellite genotyping

479 software such as Megasat (Zhan et al., 2017) which focuses on amplicon length or Micness (Suez et al.,

480 2016) which estimates the number of repeated motifs while accounting for up to one substitution within

481 the microsatellites motif. The haplotype approach has several advantages. Firstly, it is relatively

482 insensitive to sequencing error because the analysis focused on unique sequence with high coverage

483 and thus does not consider the noise generated by sequencing error which produce numerous unique 
484 low coverage sequences that are removed. Secondly, by analysing unique sequences, it accounts for any 485 kind of polymorphisms, while at the same time includes an algorithm to identify stutters resulting from 486 slippage mutation due to microsatellite instability during PCR amplification.

487 Previous studies have demonstrated the added benefit of analysing different types of polymorphism 488 within sequences compared to using amplicon size only (as in traditional capillary electrophoresis-based 489 genotyping) to differentiate alleles (Darby et al., 2016; Neophytou et al., 2018; Barbian et al., 2018; Tibihika et al., 2018; Curto et al., 2019). Size homoplasy, due to alleles identical by size but not by sequence, ranges from to 32\% and 64\% (Darby et al., 2016; Vartia et al., 2016; Barbian et al., 2018; Šarhanová et al., 2018). Here, we found high variability in size homoplasy ranging from $6 \%$ to $53 \%$ between the studied species in direct correlation to the different types of variation observed across species. SNP in the repeat motif or in the flanking region was the main source of size homoplasy which showed high prevalence in $A$. ostoyae (SNP represented $62.4 \%$ of the detected variation and $53 \%$ of size homoplasy) and to a lesser extent in Quercus sp. (SNP represented $16.8 \%$ of the detected variation and $35 \%$ of apparent homoplasy). Even for species with lower apparent polymorphism levels, such as $M$. variegatipes (6\%), S. salar (11\%) and Alosa sp. (16\%), the increase in the observed number of alleles will substantially improve genotypic resolution power (Darby et al., 2016; De Barba et al., 2016). Haplotypebased analysis that accounts for all linked variations across the whole sequence will make the most of the information available from sequence data (Barthe et al., 2012).

502 Finally, the ability to detect different sources of variation originating from several mutation mechanisms occurring at different rates provides renewed opportunities to study ecological and evolutionary events that occur at different timescales (Ramakrishnan \& Mountain, 2004; Barthe et al., 2012). Combining information on linked microsatellites and SNP (into a system called SNPSTR (Mountain et al., 2002) or HapSTR (Hey et al., 2004; Sorenson \& Dacosta, 2011)) was demonstrated to be a promising approach thanks to the increased phylogenetic resolution offered by explicitly considering complementary mutation properties of the markers. While theoretical and analytical implications of these approaches have been derived (Ramakrishnan \& Mountain, 2004; Hey et al., 2004; Payseur \& Cutter, 2006), empirical applications remain scarce and restricted to a very small number of systems due to the previous difficulties encountered to generate such empirical data (Mountain et al., 2002; Hey et al.,

512 2004). This early limitation does not hold anymore with the generalisation of sequence-based

513 microsatellite genotyping, as proposed herein, and the new ability to analyse linked microsatellites and 514 SNP as haplotype. In addition, the flexibility of coalescent programs to simulate linked loci of different 
515 types (e.g. fastsimcoal2 (Excoffier et al., 2013)) will authorize far more realistic simulation of mutation

516 mechanisms specifically tailored to each marker system. Such improvement, especially when applied to

517 tens of loci, would make simulation-based inference (Beaumont, Zhang \& Balding, 2002) more accurate

518 over an extended timescale range even for complex evolutionary history scenarios (Mountain et al.,

519 2002).

520 In conclusion, this study proposes an integrated approach to expedite the development of SSRseq

521 protocol for non-model species and provides several recommendations to improve development

522 efficiency. The two most important advices are to optimize marker selection and primer design for

523 effective multiplex PCR amplification and sequence interpretability, and to use repeated individuals to

524 assess the quality of the generated genotypic data. The ability of SSRseq to characterize SNP and indel

525 present along the sequences, in addition to the targeted microsatellite, represents a new opportunity to

526 produce empirical data to apply existing theoretical and statistical frameworks that integrate linked

527 polymorphism with different mutation characteristics (Payseur \& Cutter, 2006). Genotyping relying on

528 sequence data that are easier to normalize than traditional capillary electrophoresis genotyping through

529 automated bioinformatics pipelines will facilitate sharing of data between laboratories and incrementing

530 genotypic database that are paramount for applications in wildlife monitoring (Bradbury et al., 2018;

531 Layton et al., 2020) or agronomical research (Li et al., 2017; Yang et al., 2019). Finally, the ease of

532 parallel development for multiple species make these approach convenient to develop powerful

533 multilocus datasets for comparative population and community genetics studies (Crutsinger, 2016), and

534 to further investigate the functional implications (Bagshaw, 2017) and adaptive potential of

535 microsatellite variation among natural populations (Xie et al., 2019).

536

\section{Acknowledgments}

538 Technical developments and sequencing were performed at the Genome Transcriptome Facility of 539 Bordeaux. We thank François Meurgey for the sampling of Melipona sp. individuals. .

540

\section{References:}

542 Anvar SY, Van Der Gaag KJ, Van Der Heijden JWF, Veltrop MHAM, Vossen RHAM, De Leeuw RH, Breukel

C, Buermans HPJ, Verbeek JS, De Knijff P, Den Dunnen JT, Laros JFJ. 2014. TSSV: A tool for characterization of complex allelic variants in pure and mixed genomes. Bioinformatics 30:16511659. DOI: 10.1093/bioinformatics/btu068.

Bacles CFE, Bouchard C, Lange F, Manicki A, Tentelier C, Lepais O. 2018. Estimating the effective number of breeders from single parr samples for conservation monitoring of wild populations of Atlantic 
salmon Salmo salar. Journal of Fish Biology 92:699-726. DOI: 10.1111/jfb.13537.

Bagshaw ATM. 2017. Functional mechanisms of microsatellite DNA in eukaryotic genomes. Genome Biology and Evolution 9:2428-2443. DOI: 10.1093/gbe/evx164.

De Barba M, Miquel C, Lobréaux S, Quenette PY, Swenson JE, Taberlet P. 2016. High-throughput microsatellite genotyping in ecology: Improved accuracy, efficiency, standardization and success with low-quantity and degraded DNA. Molecular Ecology Resources 17:492-507. DOI: 10.1111/1755-0998.12594.

Barbian HJ, Connell AJ, Avitto AN, Russell RM, Smith AG, Gundlapally MS, Shazad AL, Li Y, Bibollet-Ruche F, Wroblewski EE, Mjungu D, Lonsdorf E V., Stewart FA, Piel AK, Pusey AE, Sharp PM, Hahn BH. 2018. CHIIMP: An automated high-throughput microsatellite genotyping platform reveals greater allelic diversity in wild chimpanzees. Ecology and Evolution 8:7946-7963. DOI: 10.1002/ece3.4302.

Barthe S, Gugerli F, Barkley NA, Maggia L, Cardi C, Scotti I. 2012. Always look on both sides: phylogenetic information conveyed by simple sequence repeat allele sequences. PLoS ONE 7:e40699. DOI: 10.1371/journal.pone.0040699.

Beaumont MA, Zhang W, Balding DJ. 2002. Approximate bayesian computation in population genetics. Genetics 162:2025-2035.

Blacket MJ, Robin C, Good RT, Lee SF, Miller AD. 2012. Universal primers for fluorescent labelling of PCR fragments - an efficient and cost-effective approach to genotyping by fluorescence. Molecular Ecology 12:456-463. DOI: 10.1111/j.1755-0998.2011.03104.x.

Bradbury IR, Wringe BF, Watson B, Paterson I, Horne J, Beiko R, Lehnert SJ, Clément M, Anderson EC, Jeffery NW, Duffy S, Sylvester E, Robertson M, Bentzen P. 2018. Genotyping-by-sequencing of genome-wide microsatellite loci reveals fine-scale harvest composition in a coastal Atlantic salmon fishery. Evolutionary Applications 11:918-930. DOI: 10.1111/eva.12606.

Brown SS, Chen Y-W, Wang M, Clipson A, Ochoa E, Du M-Q. 2017. PrimerPooler: automated primer pooling to prepare library for targeted sequencing. Biology Methods and Protocols 2:1-10. DOI: 10.1093/biomethods/bpx006.

Campbell NR, Harmon S, Narum SR. 2015. Genotyping-in-Thousands by sequencing (GT-seq): A cost effective SNP genotyping method based on custom amplicon sequencing. Molecular Ecology Resources 15:855-867. DOI: 10.1111/1755-0998.12357.

Castoe TA, Poole AW, Gu W, Jason de Koning AP, Daza JM, Smith EN, Pollock DD. 2010. Rapid identification of thousands of copperhead snake (Agkistrodon contortrix) microsatellite loci from modest amounts of 454 shotgun genome sequence. Molecular Ecology Resources 10:341-347. DOI: 10.1111/j.1755-0998.2009.02750.x.

Chen K, Zhou Y xun, Li K, Qi L xin, Zhang Q fei, Wang M chun, Xiao J hua. 2016. A novel three-round multiplex PCR for SNP genotyping with next generation sequencing. Analytical and Bioanalytical Chemistry:1-7. DOI: 10.1007/s00216-016-9536-6.

Crutsinger GM. 2016. A community genetics perspective: Opportunities for the coming decade. New Phytologist 210:65-70. DOI: 10.1111/nph.13537.

Curto M, Winter S, Seiter A, Schmid L, Scheicher K, Barthel LMF, Plass J, Meimberg H. 2019. Application of a SSR-GBS marker system on investigation of European Hedgehog species and their hybrid zone dynamics. Ecology and Evolution 9:2814-2832. DOI: 10.1002/ece3.4960.

Darby BJ, Erickson SF, Hervey SD, Ellis-Felege SN. 2016. Digital fragment analysis of short tandem repeats by high-throughput amplicon sequencing. Ecology and Evolution 6:4502-4512. DOI: 10.1002/ece3.2221.

Durand J, Bodenes C, Chancerel E, Frigerio JM, Vendramin G, Sebastiani F, Buonamici A, Gailing O, Koelewijn HP, Villani F, Mattioni C, Cherubini M, Goicoechea P, Herran A, Ikaran Z, Cabane C, Ueno S, Alberto F, Dumoulin PY, Guichoux E, Daruvar A de, Kremer A, Plomion C. 2010. A fast and costeffective approach to develop and map EST-SSR markers: oak as a case study. BMC Genomics 11. 
596

597

598

599

600

601

602

603

604

605

606

607

608

609

610

611

612

613

614

615

616

617

618

619

620

621

622

623

624

625

626

627

628

629

630

631

632

633

634

635

636

637

638

639

640

641

642

643

DOI: 10.1186/1471-2164-11-570.

Ellis JS, Gilbey J, Armstrong A, Balstad T, Cauwelier E, Cherbonnel C, Consuegra S, Coughlan J, Cross TF, Crozier W, Dillane E, Ensing D, García de Leániz C, García-Vázquez E, Griffiths AM, Hindar K, Hjorleifsdottir S, Knox D, Machado-Schiaffino G, McGinnity P, Meldrup D, Nielsen EE, Olafsson K, Primmer CR, Prodohl P, Stradmeyer L, Vähä J-P, Verspoor E, Wennevik V, Stevens JR. 2011. Microsatellite standardization and evaluation of genotyping error in a large multi-partner research programme for conservation of Atlantic salmon (Salmo salar L.). Genetica 139:353-367. DOI: 10.1007/s10709-011-9554-4.

Estoup A, Jarne P, Cornuet J-M. 2002. Homoplasy and mutation model at microsatellite loci and their consequences for population genetics analysis. Molecular Ecology 11:1591-1604. DOI: 10.1046/j.1365-294X.2002.01576.x.

Excoffier L, Dupanloup I, Huerta-Sánchez E, Sousa VC, Foll M. 2013. Robust demographic inference from genomic and SNP data. PLoS Genetics 9:e1003905. DOI: 10.1371/journal.pgen.1003905.

Farrell ED, Carlsson JEL, Carlsson J, Farrell ED. 2016. Next Gen Pop Gen : implementing a highthroughput approach to population genetics in boarfish (Capros aper). Royal Society Open Science 3:160651. DOI: http://dx.doi.org/10.1098/rsos.160651.

Gilbey J, Verspoor E, McLay A, Houlihan D. 2004. A microsatellite linkage map for Atlantic salmon ( Salmo salar ). Animal Genetics 35:98-105. DOI: 10.1111/j.1365-2052.2004.01091.x.

Guichoux E, Lagache L, Wagner S, Chaumeil P, Léger P, Lepais O, Lepoittevin C, Malausa T, Revardel E, Salin F, Petit RJ. 2011. Current trends in microsatellite genotyping. Molecular Ecology Resources 11:591-611. DOI: 10.1111/j.1755-0998.2011.03014.x.

Gymrek M. 2017. A genomic view of short tandem repeats. Current Opinion in Genetics \& Development 44:9-16. DOI: 10.1016/j.gde.2017.01.012.

HaasI RJ, Payseur BA. 2011. Multi-locus inference of population structure: a comparison between single nucleotide polymorphisms and microsatellites. Heredity 106:158-171. DOI: 10.1038/hdy.2010.21.

Hannan AJ. 2018. Tandem repeats mediating genetic plasticity in health and disease. Nature Reviews Genetics 19:286-298. DOI: 10.1038/nrg.2017.115.

Harrison HB, Saenz-Agudelo P, Planes S, Jones GP, Berumen ML. 2013. Relative accuracy of three common methods of parentage analysis in natural populations. Molecular Ecology 22:1158-1170. DOI: $10.1111 / \mathrm{mec} .12138$.

Hey J, Won Y-J, Sivasundar A, Nielsen R, Markert JA. 2004. Using nuclear haplotypes with microsatellites to study gene flow between recently separated Cichlid species. Molecular Ecology 13:909-919. DOI: 10.1046/j.1365-294X.2003.02031.x.

Hoffman Jl, Amos W. 2005. Microsatellite genotyping errors: detection approaches, common sources and consequences for paternal exclusion. Molecular Ecology 14:599-612. DOI: 10.1111/j.1365294X.2004.02419.x.

Holleley CE, Geerts PG. 2009. Multiplex Manager 1.0: a cross-platform computer program that plans and optimizes multiplex PCR. BioTechniques 46:511-517. DOI: 10.2144/000113156.

Hoogenboom J, de Knijff P, Laros JFJ, de Leeuw RH, van der Gaag KJ, Sijen T. 2016. FDSTools: A software package for analysis of massively parallel sequencing data with the ability to recognise and correct STR stutter and other PCR or sequencing noise. Forensic Science International: Genetics 27:27-40. DOI: 10.1016/j.fsigen.2016.11.007.

Kampfer S, Lexer C, Glössl J, Steinkellner H. 1998. Characterization of (GA)n microsatellite loci from Quercus robur. Hereditas 129:183-186. DOI: 10.1111/j.1601-5223.1998.00183.x.

King TL, Eackles MS, Letcher BH. 2005. Microsatellite DNA markers for the study of Atlantic salmon (Salmo salar) kinship, population structure, and mixed-fishery analyses. Molecular Ecology Notes 5:130-132. DOI: 10.1111/j.1471-8286.2005.00860.x.

Langmead B, Salzberg SL. 2012. Fast gapped-read alignment with Bowtie 2. Nature Methods 9:357-359.

Peer] reviewing PDF | (2019:12:44260:1:1:NEW 14 Mar 2020) 
644

645

646

647

648

649

650

651

652

653

654

655

656

657

658

659

660

661

662

663

664

665

666

667

668

669

670

671

672

673

674

675

676

677

678

679

680

681

682

683

684

685

686

687

688

689

690

691

DOI: $10.1038 /$ nmeth.1923.

Layton KKS, Dempson B, Snelgrove PVR, Duffy SJ, Messmer AM, Paterson IG, Jeffery NW, Kess T, Horne JB, Salisbury SJ, Ruzzante DE, Bentzen P, Côté D, Nugent CM, Ferguson MM, Leong JS, Koop BF, Bradbury IR. 2020. Resolving fine-scale population structure and fishery exploitation using sequenced microsatellites in a northern fish. Evolutionary Applications:eva.12922. DOI: 10.1111/eva.12922.

Lepais O, Bacles CFE. 2011. Comparison of random and SSR-enriched shotgun pyrosequencing for microsatellite discovery and single multiplex PCR optimization in Acacia harpophylla F. Muell. Ex Benth. Molecular Ecology Resources:711-724. DOI: 10.1111/j.1755-0998.2011.03002.x.

Lepais O, Manicki A, Glise S, Buoro M, Bardonnet A. 2017. Genetic architecture of threshold reaction norms for male alternative reproductive tactics in Atlantic salmon (Salmo salar L.). Scientific reports 7:43552. DOI: 10.1038/srep43552.

Li L, Fang Z, Zhou J, Chen H, Hu Z, Gao L, Chen L, Ren S, Ma H, Lu L, Zhang W, Peng H. 2017. An accurate and efficient method for large-scale SSR genotyping and applications. Nucleic Acids Research 45:e88-e88. DOI: 10.1093/nar/gkx093.

Malausa T, Gilles A, Meglécz E, Blanquart H, Duthoy S, Costedoat C, Dubut V, Pech N, CastagnoneSereno P, Délye C, Feau N, Frey P, Gauthier P, Guillemaud T, Hazard L, Le Corre V, Lung-Escarmant B, Malé P-JG, Ferreira S, Martin J-F. 2011. High-throughput microsatellite isolation through 454 GSFLX Titanium pyrosequencing of enriched DNA libraries. Molecular Ecology Resources 11:638-644. DOI: 10.1111/j.1755-0998.2011.02992.x.

Margulies M, Egholm M, Altman WE, Attiya S, Bader JS, Bemben LA, Berka J, Braverman MS, Chen Y-J, Chen Z, Dewell SB, Du L, Fierro JM, Gomes X V., Godwin BC, He W, Helgesen S, Ho CH, Irzyk GP, Jando SC, Alenquer MLI, Jarvie TP, Jirage KB, Kim J-B, Knight JR, Lanza JR, Leamon JH, Lefkowitz SM, Lei M, Li J, Lohman KL, Lu H, Makhijani VB, McDade KE, McKenna MP, Myers EW, Nickerson E, Nobile JR, Plant R, Puc BP, Ronan MT, Roth GT, Sarkis GJ, Simons JF, Simpson JW, Srinivasan M, Tartaro KR, Tomasz A, Vogt KA, Volkmer GA, Wang SH, Wang Y, Weiner MP, Yu P, Begley RF, Rothberg JM. 2005. Genome sequencing in microfabricated high-density picolitre reactors. Nature 437:376-380. DOI: 10.1038/nature03959.

Martin M. 2011. Cutadapt removes adapter sequences from high-throughput sequencing reads. EMBnet.journal 17:10. DOI: 10.14806/ej.17.1.200.

Meglécz E, Costedoat C, Dubut V, Gilles A, Malausa T, Pech N, Martin J-F. 2010. QDD: a user-friendly program to select microsatellite markers and design primers from large sequencing projects. Bioinformatics 26:403-4. DOI: 10.1093/bioinformatics/btp670.

Meglécz E, Pech N, Gilles A, Dubut V, Hingamp P, Trilles A, Grenier R, Martin J-F. 2014. QDD version 3.1: a user-friendly computer program for microsatellite selection and primer design revisited: experimental validation of variables determining genotyping success rate. Molecular Ecology Resources 14:1302-1313. DOI: 10.1111/1755-0998.12271.

Moran P, Teel DJ, LaHood ES, Drake J, Kalinowski S. 2006. Standardising multi-laboratory microsatellite data in Pacific salmon: an historical view of the future. Ecology of Freshwater Fish 15:597-605. DOI: 10.1111/j.1600-0633.2006.00201.x.

Mountain JL, Knight A, Jobin M, Gignoux C, Miller A, Lin AA, Underhill PA. 2002. SNPSTRs: Empirically derived, rapidly typed, autosomal haplotypes for inference of population history and mutational processes. Genome Research 12:1766-1772. DOI: 10.1101/gr.238602.

Neophytou C, Torutaeva E, Winter S, Meimberg H, Hasenauer H, Curto M. 2018. Analysis of microsatellite loci in tree of heaven (Ailanthus altissima (Mill.) Swingle) using SSR-GBS. Tree Genetics and Genomes 14. DOI: 10.1007/s11295-018-1295-4.

O'Reilly P, Wright JM. 1995. The evolving technology of DNA fingerprinting and its application to fisheries and aquaculture. Journal of Fish Biology 47:29-55. DOI: 10.1111/j.1095- 
692

693

694

695

696

697

698

699

700

701

702

703

704

705

706

707

708

709

710

711

712

713

714

715

716

717

718

719

720

721

722

723

724

725

726

727

728

729

730

731

732

733

734

735

736

737

738

739

8649.1995.tb06042.x.

Ozaki A, Sakamoto T, Khoo S, Nakamura K, Coimbra MRM, Akutsu T, Okamoto N. 2001. Quantitative trait loci (QTLs) associated with resistance/susceptibility to infectious pancreatic necrosis virus (IPNV) in rainbow trout (Oncorhynchus mykiss). Molecular Genetics and Genomics 265:23-31. DOI: 10.1007/s004380000392.

Paterson S, Piertney SB, Knox D, Gilbey J, Verspoor E. 2004. Characterization and PCR multiplexing of novel highly variable tetranucleotide Atlantic salmon (Salmo salar L.) microsatellites. Molecular Ecology Notes 4:160-162. DOI: 10.1111/j.1471-8286.2004.00598.x.

Payseur BA, Cutter AD. 2006. Integrating patterns of polymorphism at SNPs and STRs. Trends in Genetics 22:424-429. DOI: 10.1016/j.tig.2006.06.009.

Pimentel JSM, Carmo AO, Rosse IC, Martins APV, Ludwig S, Facchin S, Pereira AH, Brandão-Dias PFP, Abreu NL, Kalapothakis E. 2018. High-throughput sequencing strategy for microsatellite genotyping using neotropical fish as a model. Frontiers in Genetics 9:73. DOI: 10.3389/fgene.2018.00073.

Plomion C, Aury JM, Amselem J, Leroy T, Murat F, Duplessis S, Faye S, Francillonne N, Labadie K, Le Provost G, Lesur I, Bartholomé J, Faivre-Rampant P, Kohler A, Leplé JC, Chantret N, Chen J, Diévart $A$, Alaeitabar $T$, Barbe $V$, Belser $C$, Bergès $H$, Bodénès $C$, Bogeat-Triboulot $M B$, Bouffaud $M L$, Brachi B, Chancerel E, Cohen D, Couloux A, Da Silva C, Dossat C, Ehrenmann F, Gaspin C, Grima-Pettenati J, Guichoux E, Hecker A, Herrmann S, Hugueney P, Hummel I, Klopp C, Lalanne C, Lascoux M, Lasserre E, Lemainque A, Desprez-Loustau ML, Luyten I, Madoui MA, Mangenot S, Marchal C, Maumus F, Mercier J, Michotey C, Panaud O, Picault N, Rouhier N, Rué O, Rustenholz C, Salin F, Soler M, Tarkka M, Velt A, Zanne AE, Martin F, Wincker P, Quesneville H, Kremer A, Salse J. 2018. Oak genome reveals facets of long lifespan. Nature Plants 4:440-452. DOI: 10.1038/s41477-0180172-3.

Pompanon F, Bonin A, Bellemain E, Taberlet P. 2005. Genotyping errors: causes, consequences and solutions. Nature reviews. Genetics 6:847-59. DOI: 10.1038/nrg1707.

Quinlan AR. 2014. BEDTools: The Swiss-Army Tool for Genome Feature Analysis. Current Protocols in Bioinformatics 47:11.12.1-11.12.34. DOI: 10.1002/0471250953.bi1112s47.

Ramakrishnan U, Mountain JL. 2004. Precision and accuracy of divergence time estimates from STR and SNPSTR variation. Molecular Biology and Evolution 21:1960-1971. DOI: 10.1093/molbev/msh212.

Rexroad CE, Coleman RL, Martin AM, Hershberger WK, Killefer J. 2001. Thirty-five polymorphic microsatellite markers for rainbow trout (Oncorhynchus mykiss). Animal Genetics 32:317-319. DOI: 10.1046/j.1365-2052.2001.0730b.x.

Rougemont $Q$, Besnard AL, Baglinière JL, Launey S. 2015. Characterization of thirteen new microsatellite markers for allis shad (Alosa alosa) and twaite shad (Alosa fallax). Conservation Genetics Resources 7:259-261. DOI: 10.1007/s12686-014-0352-z.

Sadd BM, Schaack S, Zhao X, Sun C, Su L. 2018. Tandem repeats contribute to coding sequence variation in bumblebees (Hymenoptera: Apidae). Genome Biology and Evolution 10:3176-3187. DOI: 10.1093/gbe/evy244.

Šarhanová P, Pfanzelt S, Brandt R, Himmelbach A, Blattner FR. 2018. SSR-seq: Genotyping of microsatellites using next-generation sequencing reveals higher level of polymorphism as compared to traditional fragment size scoring. Ecology and Evolution 8:10817-10833. DOI: 10.1002/ece3.4533.

Schloss PD, Westcott SL, Ryabin T, Hall JR, Hartmann M, Hollister EB, Lesniewski RA, Oakley BB, Parks DH, Robinson CJ, Sahl JW, Stres B, Thallinger GG, Van Horn DJ, Weber CF. 2009. Introducing mothur: open-source, platform-independent, community-supported software for describing and comparing microbial communities. Applied and environmental microbiology 75:7537-41. DOI: 10.1128/AEM.01541-09.

Schlötterer C. 2004. The evolution of molecular markers - just a matter of fashion? Nature Reviews

Peer] reviewing PDF | (2019:12:44260:1:1:NEW 14 Mar 2020) 
Genetics 5:63-69. DOI: 10.1038/nrg1249.

Seeb JE, Carvalho G, Hauser L, Naish K, Roberts S, Seeb LW. 2011. Single-nucleotide polymorphism (SNP) discovery and applications of SNP genotyping in nonmodel organisms. Molecular Ecology Resources 11:1-8. DOI: 10.1111/j.1755-0998.2010.02979.x.

Sipos G, Prasanna AN, Walter MC, O’Connor E, Bálint B, Krizsán K, Kiss B, Hess J, Varga T, Slot J, Riley R, Bóka B, Rigling D, Barry K, Lee J, Mihaltcheva S, LaButti K, Lipzen A, Waldron R, Moloney NM, Sperisen C, Kredics L, Vágvölgyi C, Patrignani A, Fitzpatrick D, Nagy I, Doyle S, Anderson JB, Grigoriev I V., Güldener U, Münsterkötter M, Nagy LG. 2017. Genome expansion and lineagespecific genetic innovations in the forest pathogenic fungi Armillaria. Nature Ecology \& Evolution 1:1931-1941. DOI: 10.1038/s41559-017-0347-8.

Slettan A, Olsaker I, Lie $\varnothing$. 1996. Polymorphic Atlantic salmon, Salmo salar L., microsatellites at the SSOSL438, SSOSL439 and SSOSL444 loci. Animal Genetics 27:57-58. DOI: 10.1111/j.13652052.1996.tb01180.x.

Sorenson MD, Dacosta JM. 2011. Genotyping HapSTR loci: phase determination from direct sequencing of PCR products. Molecular Ecology Resources 11:1068-1075. DOI: 10.1111/j.17550998.2011.03036.x.

Steinkellner H, Fluch S, Turetschek E, Lexer C, Streiff R, Kremer A, Burg K, Glössl J. 1997. Identification and characterization of (GA/CT)n microsatellite loci from Quercus petraea. Plant Molecular Biology 33:1093-1096. DOI: 10.1023/A:1005736722794.

Suez M, Behdenna A, Brouillet S, Graça P, Higuet D, Achaz G. 2016. MicNeSs: Genotyping microsatellite loci from a collection of (NGS) reads. Molecular Ecology Resources 16:524-533. DOI: 10.1111/17550998.12467.

Thorsen J, Zhu B, Frengen E, Osoegawa K, de Jong PJ, Koop BF, Davidson WS, Høyheim B. 2005. A highly redundant BAC library of Atlantic salmon (Salmo salar): an important tool for salmon projects. $B M C$ Genomics 6:50. DOI: 10.1186/1471-2164-6-50.

Tibihika PD, Curto M, Dornstauder-Schrammel E, Winter S, Alemayehu E, Waidbacher H, Meimberg H. 2018. Application of microsatellite genotyping by sequencing (SSR-GBS) to measure genetic diversity of the East African Oreochromis niloticus. Conservation Genetics:1-16. DOI: 10.1007/s10592-018-1136-x.

Vartia S, Villanueva-Cañas JL, Finarelli J, Farrell ED, Collins PC, Hughes GM, Carlsson JEL, Gauthier DT, McGinnity P, Cross TF, FitzGerald RD, Mirimin L, Crispie F, Cotter PD, Carlsson J. 2016. A novel method of microsatellite genotyping-by-sequencing using individual combinatorial barcoding. Royal Society Open Science 3:150565. DOI: 10.1098/rsos.150565.

Vasemägi A, Nilsson J, Primmer CR. 2005. Expressed sequence tag-linked microsatellites as a source of gene-associated polymorphisms for detecting signatures of divergent selection in Atlantic salmon (Salmo salar L.). Molecular Biology and Evolution 22:1067-76. DOI: 10.1093/molbev/msi093.

Viard F, Franck P, Dubois MP, Estoup A, Jarne P. 1998. Variation of microsatellite size homoplasy across electromorphs, loci, and populations in three invertebrate species. Journal of molecular evolution 47:42-51.

Viruel J, Haguenauer A, Juin M, Mirleau F, Bouteiller D, Boudagher-Kharrat M, Ouahmane L, La Malfa S, Médail F, Sanguin H, Nieto Feliner G, Baumel A. 2018. Advances in genotyping microsatellite markers through sequencing and consequences of scoring methods for Ceratonia siliqua (Leguminosae). Applications in plant sciences 6:e01201. DOI: 10.1002/aps3.1201.

Wang J. 2017. Estimating genotyping errors from genotype and reconstructed pedigree data. Methods in Ecology and Evolution Early View. DOI: 10.1111/2041-210X.12859.

Willems T, Gymrek M, Highnam G, 1000 Genomes Project Consortium T 1000 GP, Mittelman D, Erlich Y. 2014. The landscape of human STR variation. Genome research 24:1894-904. DOI: 10.1101/gr.177774.114. 
802

803

804

805

806

807

808

809

810

811

Xie KT, Wang G, Thompson AC, Wucherpfennig JI, Reimchen TE, MacColl ADC, Schluter D, Bell MA, Vasquez KM, Kingsley DM. 2019. DNA fragility in the parallel evolution of pelvic reduction in stickleback fish. Science 363:81-84. DOI: 10.1126/science.aan1425.

Yang J, Zhang J, Han R, Zhang F, Mao A, Luo J, Dong B, Liu H, Tang H, Zhang J, Wen C. 2019. Target SSRSeq: A Novel SSR Genotyping Technology Associate With Perfect SSRs in Genetic Analysis of Cucumber Varieties. Frontiers in Plant Science 10:531. DOI: 10.3389/fpls.2019.00531.

Yano A, Nicol B, Jouanno E, Quillet E, Fostier A, Guyomard R, Guigen Y. 2013. The sexually dimorphic on the $\mathrm{Y}$-chromosome gene (sdY) is a conserved male-specific $\mathrm{Y}$-chromosome sequence in many salmonids. Evolutionary Applications 6:486-496. DOI: 10.1111/eva.12032.

Zhan L, Paterson IG, Fraser BA, Watson B, Bradbury IR, Nadukkalam Ravindran P, Reznick D, Beiko RG, Bentzen P. 2017. megasat: Automated inference of microsatellite genotypes from sequence data. Molecular Ecology Resources 17:247-256. DOI: 10.1111/1755-0998.12561.

Zhang J, Kobert K, Flouri T, Stamatakis A. 2014. PEAR: A fast and accurate Illumina Paired-End reAd mergeR. Bioinformatics 30:614-620. DOI: 10.1093/bioinformatics/btt593.

\section{Data Accessibility Statement}

- DNA sequences: sequence reads from SSRseq run were submitted to the European Nucleotide Archive SRA under the accession number PRJEB31908 (Alosa sp.), PRJEB31909 (A. ostoyae), PRJEB31910 (M. variegatipes), PRJEB31913 (Quercus sp.) and PRJEB31914 (S. salar). Random shotgun sequencing reads from one $M$. variegatipes individual for microsatellite discovery was submitted under the accession number ERR3255838.

- Pipeline scripts for automated sequence-based microsatellite genotyping with documentation and example files are available at Data Inra https://doi.org/10.15454/HBXKVA. 


\section{Table $\mathbf{1}$ (on next page)}

SSRseq development strategy and DNA characteristics of species used in this study. 
1 Table 1: SSRseq development strategy and DNA characteristics of species used in this study.

\begin{tabular}{|c|c|c|c|c|c|c|c|}
\hline Kingdom & Class & Species & $\begin{array}{l}\text { SSRseq development } \\
\text { strategy }\end{array}$ & $\begin{array}{l}\text { Number of } \\
\text { DNA extracted } \\
\text { individuals }\end{array}$ & $\begin{array}{l}\text { DNA extraction } \\
\text { protocol }\end{array}$ & DNA quality & Reference \\
\hline Animalia & Actinopterygii & Salmo salar & Previously developed loci & 1152 & $\begin{array}{l}\text { Salt-chloroform } \\
\text { (Gauthey et al., } \\
\text { 2015) }\end{array}$ & High & $\begin{array}{l}\text { (Bacles et al., } \\
\text { 2018; Lepais } \\
\text { et al., 2017) }\end{array}$ \\
\hline Plantae & Eudicots & $\begin{array}{l}\text { Quercus } \\
\text { faginea, } \\
\text { Q.canariensis }\end{array}$ & $\begin{array}{l}\text { Re-designed primers } \\
\text { around already developed } \\
\text { loci using reference } \\
\text { genome sequence of } \\
\text { closely related species }\end{array}$ & 380 & $\begin{array}{l}\text { Invisorb DNA } \\
\text { Plant HTS } 96 \text { kit }\end{array}$ & High & this study \\
\hline Animalia & Actinopterygii & $\begin{array}{l}\text { Alosa alosa, } \\
\text { A.fallax }\end{array}$ & $\begin{array}{l}\text { De novo loci development } \\
\text { based on available } \\
\text { repeat-enriched library } \\
\text { sequencing }\end{array}$ & 382 & $\begin{array}{l}\text { Invitrogen } \\
\text { PureLink } \\
\text { Genomic DNA } \\
\text { Mini kit }\end{array}$ & $\begin{array}{l}\text { Highly } \\
\text { degraded }\end{array}$ & $\begin{array}{l}\text { (Taillebois et } \\
\text { al., submitted) }\end{array}$ \\
\hline Fungi & Agaricomycetes & $\begin{array}{l}\text { Armillaria } \\
\text { ostoyae }\end{array}$ & $\begin{array}{l}\text { De novo loci development } \\
\text { based on reference } \\
\text { genome sequence }\end{array}$ & 384 & $\begin{array}{l}\text { CTAB } \\
\text { (Prospero, Lung- } \\
\text { Escarmant, \& } \\
\text { Dutech, 2008) }\end{array}$ & Heterogeneous & this study \\
\hline
\end{tabular}

2 
Table 2 (on next page)

Summary of the tested scenarios for SSRseq genotyping. 
1 Table 2: Summary of the tested scenarios for SSRseq genotyping

\begin{tabular}{|c|c|c|c|c|c|c|c|c|c|c|c|c|}
\hline Species & $\begin{array}{l}\text { SSRseq } \\
\text { development } \\
\text { strategy }\end{array}$ & $\begin{array}{l}\text { Candidate } \\
\text { loci }\end{array}$ & $\begin{array}{l}\text { Screened } \\
\text { loci }\end{array}$ & $\begin{array}{l}\text { Number of loci in a } \\
\text { single multiplexed } \\
\text { PCR }\end{array}$ & $\begin{array}{l}\text { Sequencing } \\
\text { Plateform }\end{array}$ & $\begin{array}{l}\text { Total } \\
\text { number of } \\
\text { individuals } \\
\text { sequenced }\end{array}$ & $\begin{array}{l}\text { Number of } \\
\text { individual } \\
\text { analyzed for } \\
\text { this study }\end{array}$ & $\begin{array}{l}\text { Number of } \\
\text { repeated } \\
\text { individuals }\end{array}$ & $\begin{array}{l}\text { Sequenced } \\
\operatorname{loci}^{\dagger}\end{array}$ & $\begin{array}{l}\text { Mean (cv) } \\
\text { sequences / } \\
\text { loci / } \\
\text { individual }\end{array}$ & $\begin{array}{l}\text { Reliable } \\
\text { loci } \\
\text { genotyped }^{\ddagger}\end{array}$ & $\begin{array}{l}\text { Overall } \\
\text { success } \\
\text { rate }\end{array}$ \\
\hline \multirow[t]{2}{*}{ S. salar } & $\begin{array}{l}\text { Previously } \\
\text { developed loci }\end{array}$ & 81 & - & 23 & $\begin{array}{l}\text { Ion Torrent } \\
\text { PGM i316 }\end{array}$ & 960 & 66 & 66 & 20 & $161(61 \%)$ & 9 & $39 \%$ \\
\hline & & & & 23 & $\begin{array}{l}\text { Illumina MiSeq } \\
-1 / 2 \text { nano PE }\end{array}$ & 192 & 96 & 96 & 20 & $70(41 \%)$ & 10 & $43 \%$ \\
\hline S. salar & $\begin{array}{l}\text { Previously } \\
\text { developed loci }\end{array}$ & $15 \S$ & - & 15 & $\begin{array}{l}\text { Illumina MiSeq } \\
-1 / 2 \text { nano PE }\end{array}$ & 192 & 96 & 96 & 13 & 99 (65\%) & 7 & $47 \%$ \\
\hline Quercus sp. & $\begin{array}{l}\text { Primer } \\
\text { redesign } \\
\text { around } \\
\text { previously } \\
\text { developped loci }\end{array}$ & 462 & 60 & 60 & $\begin{array}{l}\text { Illumina MiSeq } \\
-1 / 3 \text { V2 PE }\end{array}$ & 380 & 46 & 46 & 53 & $260(32 \%)$ & 40 & $67 \%$ \\
\hline \multirow[t]{3}{*}{ Alosa sp. } & De novo & 2872 & 60 & 28 & $\begin{array}{l}\text { Illumina MiSeq } \\
-1 \text { nano SE }\end{array}$ & 382 & 156 & 156 & 25 & 95 (58\%) & 21 & $75 \%$ \\
\hline & & & & 28 & $\begin{array}{l}\text { Illumina MiSeq } \\
-2 \text { nano SE }\end{array}$ & 382 & 156 & 156 & 26 & $198(58 \%)$ & 24 & $86 \%$ \\
\hline & & & & 28 & $\begin{array}{l}\text { Illumina MiSeq } \\
-3 \text { nano SE }\end{array}$ & 382 & 156 & 156 & 26 & 267 (58\%) & 24 & $86 \%$ \\
\hline A. ostoyae & De novo & 1806 & 60 & 51 & $\begin{array}{l}\text { Illumina MiSeq } \\
-1 / 2 \text { V2 PE }\end{array}$ & 384 & 384 & 96 & 48 & $243(83 \%)$ & 38 & $75 \%$ \\
\hline $\begin{array}{l}M \text {. } \\
\text { variegatipes }\end{array}$ & De novo & 8937 & 60 & 54 & $\begin{array}{l}\text { Illumina MiSeq } \\
-1 / 4 \text { V2 PE }\end{array}$ & 182 & 91 & 91 & 49 & $176(45 \%)$ & 39 & $72 \%$ \\
\hline
\end{tabular}

$2{ }^{\dagger}$ : loci showing substantial evidence for minimum sequencing success (at least 20 sequences in at least $50 \%$ of the individuals); ${ }^{\ddagger}$ : reliable loci (less than $50 \%$ of

3 missing data among individuals and less than $6 \%$ of genotyping error based on comparison of repeated genotyping); ${ }^{\S}$ routinely genotyped using optimized

4 multiplexed PCR and capillary-based sequencer. FDSTools analysis using two parameter sets: stutterfinder -s:-1:50,+1:10 allelefinder -m 15 -n 20; and

5 stuttermark -s:-1:70,+1:10 allelefinder -m 10 -n 20. For each marker, four parameter combination were used (two strategies and two parameters set) and for

6 each strategy, the best parameter set was used for a given locus. 
Table 3 (on next page)

Detected polymorphism. 
1 Table 3: Detected polymorphism.

\begin{tabular}{|c|c|c|c|c|c|c|c|c|c|c|}
\hline \multirow[b]{3}{*}{ Species } & \multirow[b]{3}{*}{$\begin{array}{l}\text { Number } \\
\text { of loci }\end{array}$} & \multirow[b]{3}{*}{$\begin{array}{l}\text { Number of alleles } \\
\text { with sequence } \\
\text { difference }^{+}\end{array}$} & \multirow[b]{3}{*}{$\begin{array}{l}\text { Number of alleles } \\
\text { differing by } \\
\text { amplicon size }\end{array}$} & \multirow[b]{3}{*}{$\begin{array}{l}\% \text { of size } \\
\text { homoplasy }\end{array}$} & \multirow[b]{3}{*}{$\begin{array}{l}\% \text { of increase } \\
\text { in alleles due } \\
\text { to sequence }\end{array}$} & \multirow{2}{*}{\multicolumn{3}{|c|}{$\begin{array}{l}\text { Polymorphism in the repeat } \\
\text { motif }^{\S}\end{array}$}} & & \\
\hline & & & & & & & & & \multicolumn{2}{|c|}{$\begin{array}{l}\text { Polymorphism in the } \\
\text { flanking sequences }^{\S}\end{array}$} \\
\hline & & & & & & $\begin{array}{l}\text { Repeat } \\
\text { number } \\
\text { variation }\end{array}$ & SNP & Indel & SNP & Indel \\
\hline S. salar & 14 & 122 & 108 & $11 \%$ & $13 \%$ & $107(14)$ & $3(3)$ & - & $2(2)$ & $3(3)$ \\
\hline Quercus sp. & 40 & 537 & 346 & $35 \%$ & $55 \%$ & $406(40)$ & $38(25)$ & $1(1)$ & $47(18)$ & $13(10)$ \\
\hline Alosa sp. & 24 & 174 & 150 & $14 \%$ & $16 \%$ & $130(23)$ & $13(15)$ & $2(2)$ & $4(4)$ & $3(3)$ \\
\hline A. ostoyae & 38 & 398 & 187 & $53 \%$ & $113 \%$ & $187(33)$ & $79(26)$ & $8(7)$ & $312(23)$ & $41(16)$ \\
\hline M. variegatipes & 39 & 166 & 156 & $6 \%$ & $6 \%$ & $147(39)$ & $3(3)$ & $1(1)$ & $9(7)$ & $5(5)$ \\
\hline
\end{tabular}

${ }^{\dagger}$ : irrespectively of polymorphism type, computed based on the Combined analysis strategy; ${ }^{\ddagger}$ : simulating the number of alleles that would have been identified using traditional capillary electrophoresis, computed based on the FullLength analysis strategy and accounting for allele size only on the same locus as the Combined approach. ${ }^{\S}$ : total number of alleles (and number of loci in brackets) for each polymorphism type. ": combination of two sequence based microsatellite genotyping protocols. 


\section{Figure 1}

Workflow for SSRseq markers optimization or development depending on genomic resource availability, from selection to multiplexed amplification and library preparation to bioinformatics analysis.

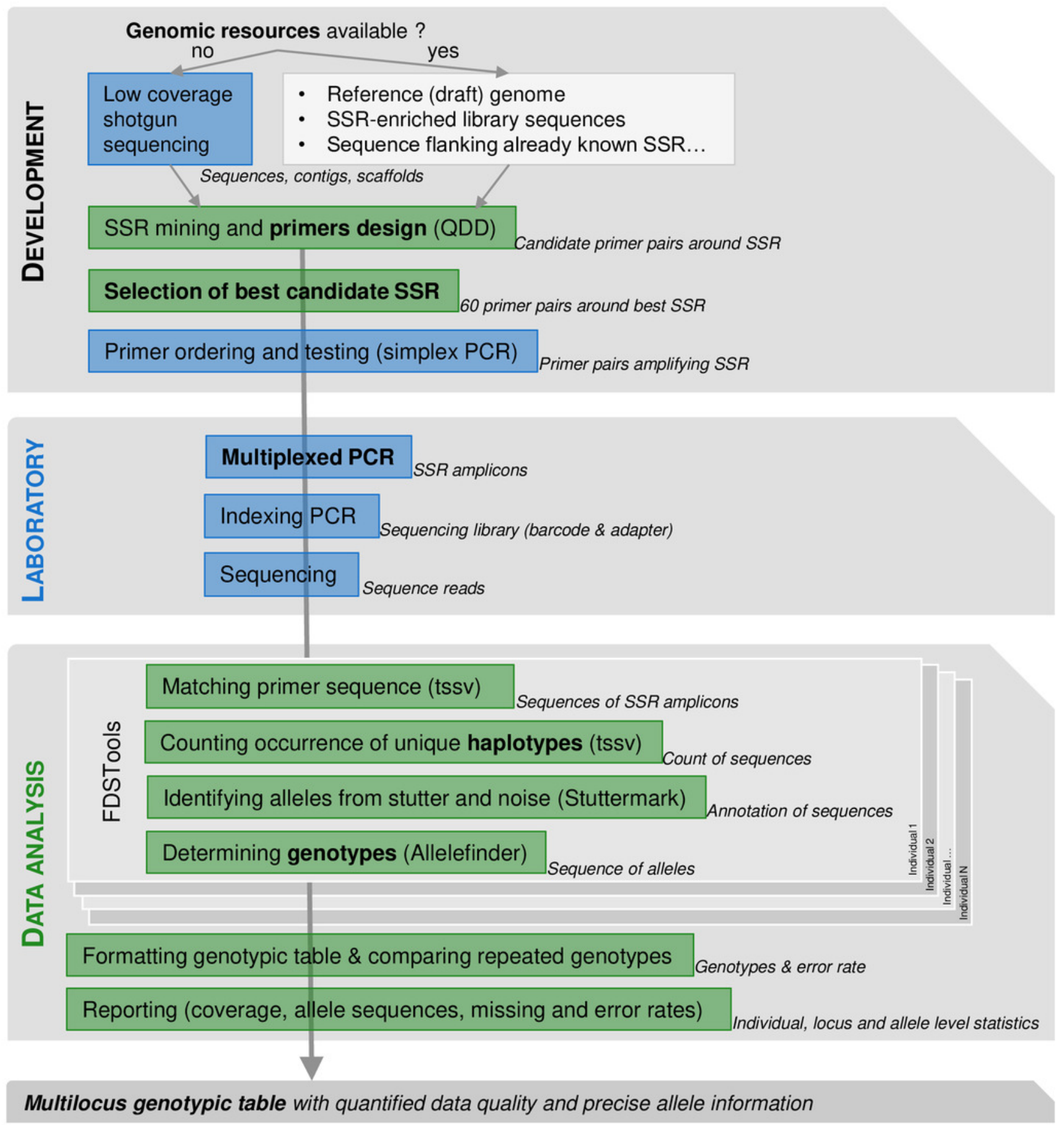




\section{Figure 2}

Results of SSRsq development from previously developed microsatellites.

S. salar for (a) a new multiplex of 23 microsatellite sequenced with Ion Torrent PGM and (b) Illumina MiSeq sequencing platforms, and (c) a routinely-used multiplex of 15 microsatellites sequenced with Illumina MiSeq sequencing platform. Number of reliable loci, total number of alleles, missing data and allelic error rates are indicated for three bioinformatics analysis strategies that focused either on all polymorphism across the sequence, on polymorphism within the repeated motif only, or a combination of the best strategy for each locus.
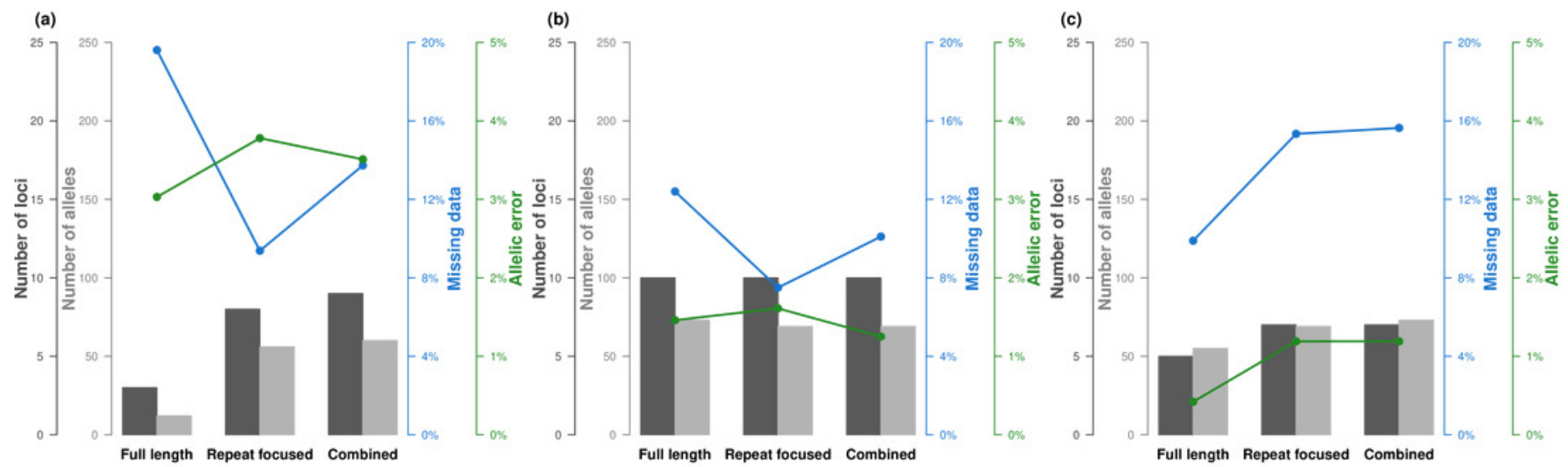


\section{Figure 3}

Results of SSRseq development based on newly optimized microsatellites.

(a) Quercus sp., (b) Alosa sp., (c) A. ostoyae and (d) M. variegatipes sequenced with Illumina MiSeq sequencing platform. Number of reliable loci, total number of alleles, missing data and allelic error rates are indicated for three bioinformatics analysis strategies that focused either on all polymorphism across the sequence, on polymorphism within the repeated motif only, or a combination of the best strategy for each locus.
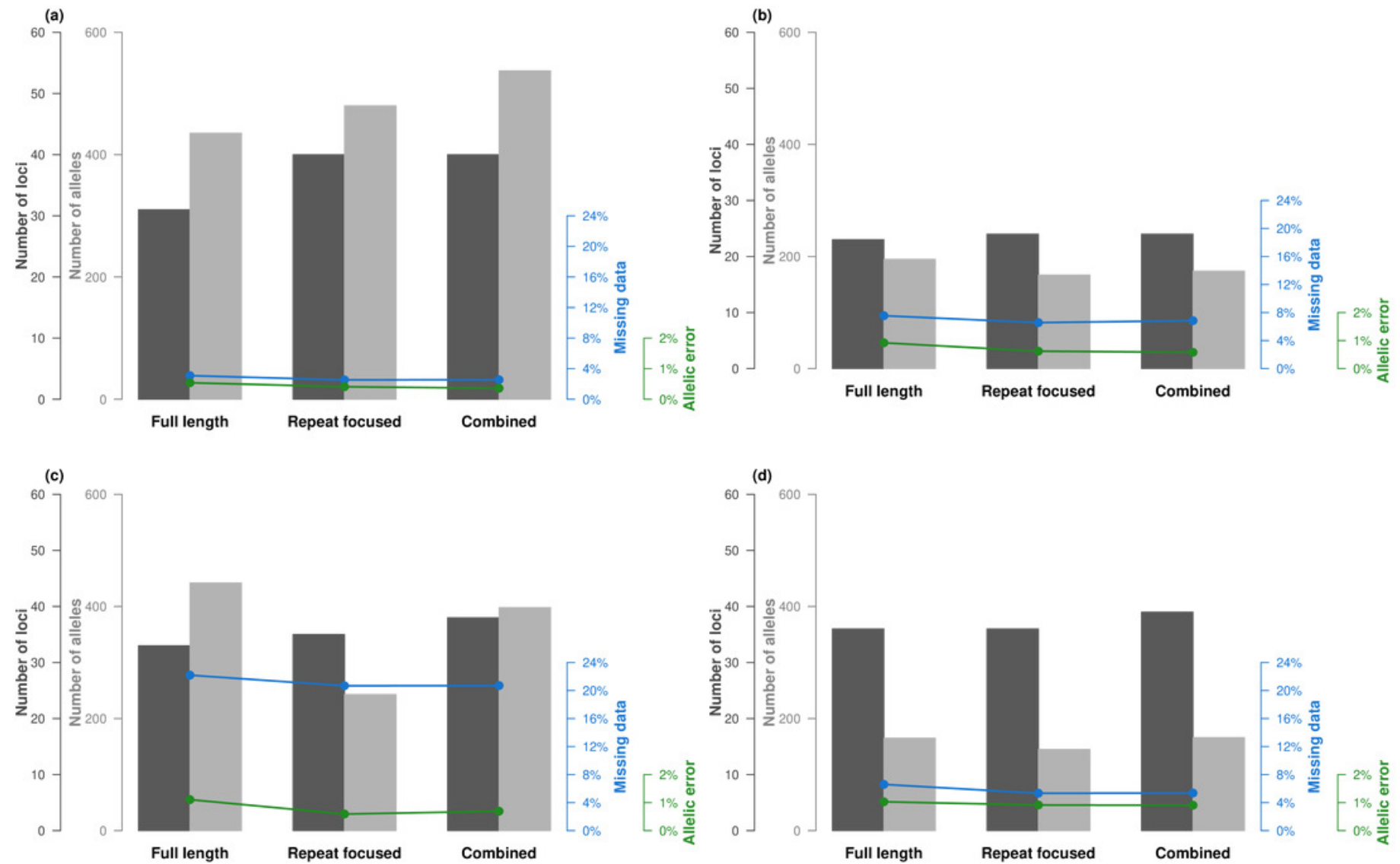
Figure 4

Proportion of detected polymorphism types within the repeat motif or in the flanking sequence for each sample per species group.
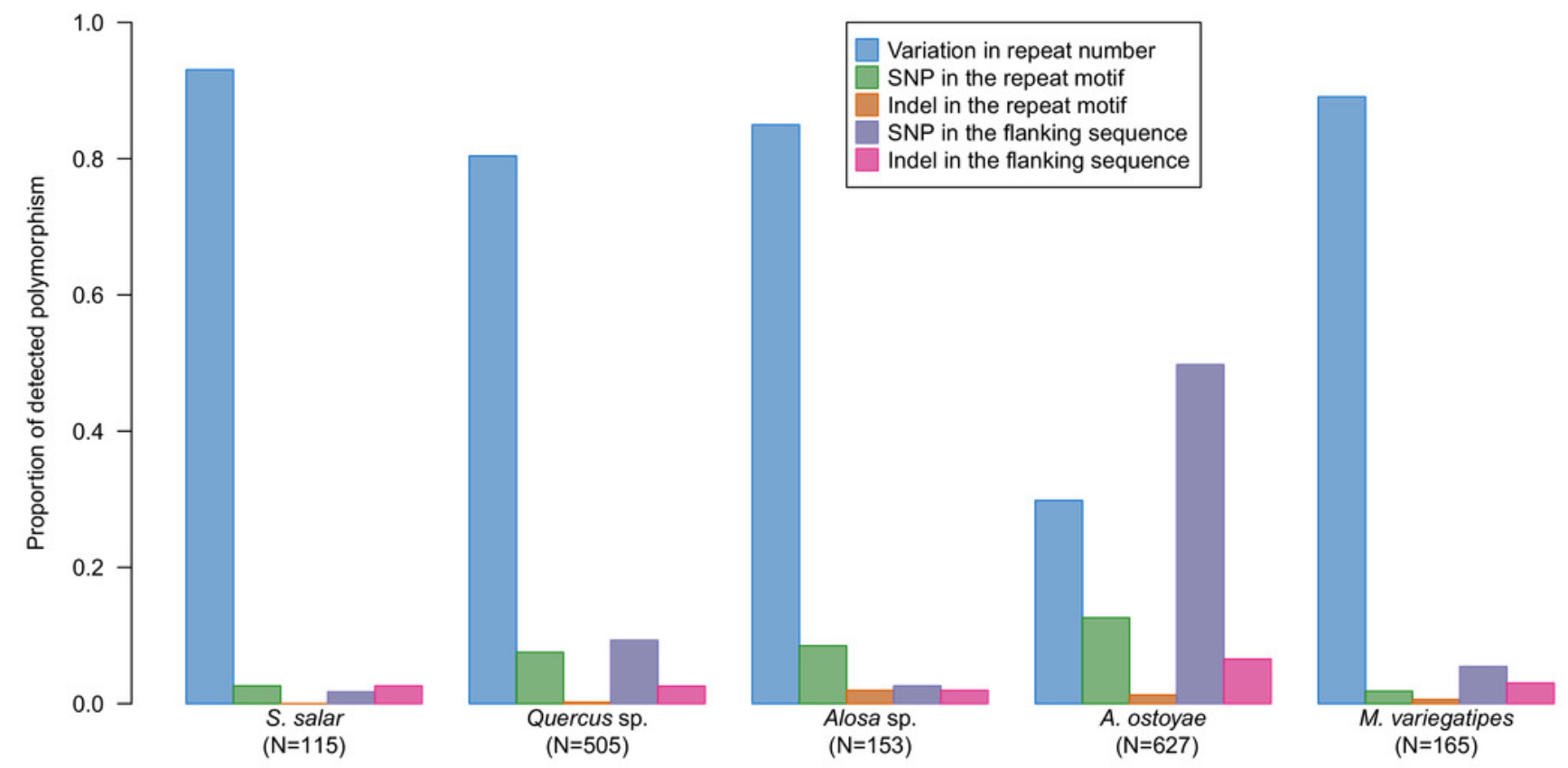